\title{
Neuronavigated percutaneous approach to the sphenopalatine ganglion
}

\author{
Nicola Benedetto, MD, PhD, and Paolo Perrini, MD, PhD \\ Neurosurgical Department, Azienda Ospedaliero Universitaria Pisana-AOUP, Pisa, Italy \\ The sphenopalatine ganglion (SPG) has been assumed to be involved in the genesis of several types of facial pain, \\ including Sluder's neuralgia, trigeminal neuralgia, persistent idiopathic facial pain, cluster headache, and atypical facial \\ pain. The gold standard treatments for SPG-related pain are percutaneous procedures performed with the aid of fluoros- \\ copy or CT. In this technical note the authors present, for the first time, an SPG approach using the aid of a neuronaviga- \\ tor. \\ http://thejns.org/doi/abs/10.3171/2016.1.JNS152272
}

KEY WORDS atypical facial pain; neuronavigation; percutaneous; sphenopalatine ganglion; trigeminal neuralgia; functional neurosurgery

$\mathrm{T}$ HE sphenopalatine ganglion (SPG) is the largest group of extracranial neurons. It contains both sympathetic and parasympathetic neurons and has been assumed to be involved in a wide spectrum of facial pain. Although the effective role of the SPG in generating head and facial pain is still under investigation, in recent decades treatment involving the SPG has improved dramatically due to advancements in neuroimaging, allowing for more precise targeting. The anatomical site of the SPG is a hard-to-reach triangular fossa-the pterygopalatine fossa (PPF) - with the base sitting laterally and the apex situated medially. The PPF becomes narrower from the base to the apex and is surrounded by the posterior wall of the maxillary sinus anteriorly, the internal pterygoid process posteriorly, and the perpendicular plate of the palatine bone medially. Several techniques have been proposed to treat SPG-related pain, ranging from endoscopic endonasal procedures ${ }^{1}$ to percutaneous procedures, ${ }^{4,5,8}$ and more recently, even stereotactic Gamma Knife (GK) treatment. ${ }^{2}$ In this technical note we present an easy way to reach the SPG percutaneously using a "navigated" needle. The neuronavigated percutaneous approach to the SPG is an attractive option in patients who are not good surgical candidates or who possibly underwent unsuccessful surgical and/or radiosurgical therapy.

\section{Operative Technique}

On the day of the procedure the $\mathrm{CT}$ scan is uploaded to the StealthStation S7 system (Medtronic) and the point between the foramen rotundum and the vidian canal is identified (Fig. 1); this point is most likely the site of the SPG and is then set as the target point. The entry point is chosen below the zygomatic arch to avoid the coronoid process of the mandibular bone (Fig. 2). The procedure is performed in the operative theater under local anesthesia.

Once the trajectory is planned, the patient is placed supine on the table with the head on a horseshoe headrest and rotated contralaterally from the affected side. A nontraumatic magnetic reference tool is placed on the patient's forehead, and the registration procedure is performed. A probe tool is then used to find the entry point, and $3 \mathrm{ml}$ of lidocaine is administrated.

To build a navigated needle that is recognizable by the navigation system, a magnetic stylet is inserted through 2 rubber plunger tips from insulin hypodermic syringes that have previously been pierced centrally. It is then introduced into an insulin syringe, while a third plunger tip is placed into the syringe at the top to prevent further displacement of the probe (Figs. 3 and 4). A 20-G spinal needle is then connected to the syringe and the distance between the tip of the needle and the tip of the probe is set as the offset on neuronavigation, thus transforming the needle into a navigated needle (Fig. 5).

Using the guidance function (Fig. 6) we follow the preplanned path, inserting the needle until we reach the target. Once there, we disconnect the insulin syringe with the probe and replace it with a syringe containing $1 \mathrm{ml}$ of

ABBREVIATIONS GK = Gamma Knife; PPF = pterygopalatine fossa; $R F=$ radiofrequency; $S P G$ = sphenopalatine ganglion; $T N=$ trigeminal neuralgia .

SUBMITTED September 28, 2015. ACCEPTED January 28, 2016.

INCLUDE WHEN CITING Published online April 22, 2016; DOI: 10.3171/2016.1.JNS152272. 

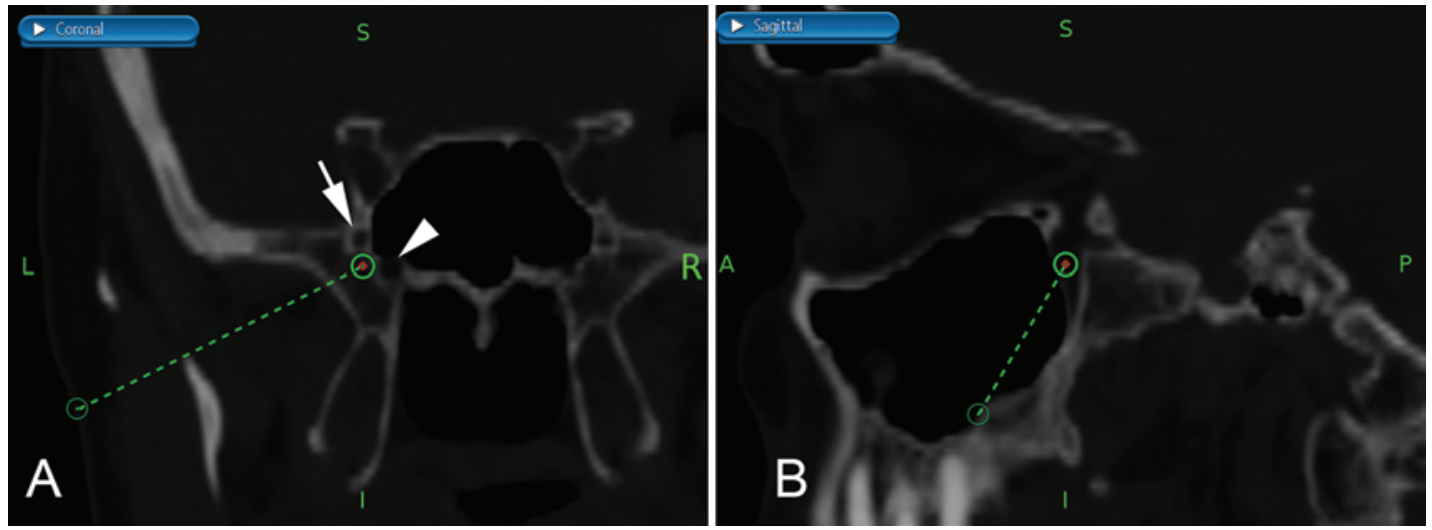

FIG. 1. Coronal (A) and sagittal (B) CT reconstruction of the PPF. The target (thick green circle) lies between the foramen rotundum (arrow) and the vidian canal (arrowhead). The thin green circle is the entry point and the dashed line is the planned trajectory. Figure is available in color online only.

lidocaine. After 10 seconds of aspiration with no signs of hemorrhage we inject the lidocaine, and then after 1 minute we follow this with $1 \mathrm{ml}$ of pure alcohol. The needle is eventually extracted. The patient is kept under observation for the following 2 hours and eventually discharged.

\section{Results}

Over the last 10 months we have treated 7 patients $(5$ females and 2 males) with a navigated percutaneous approach to the SPG. Five of these 7 patients had a history of trigeminal neuralgia (TN) that had previously been treated with microvascular decompression and at least 1 other procedure (percutaneous balloon compression, GK treatment, or partial retrogasserian rhizotomy), and the other 2 suffered from TN due to multiple sclerosis, which had been treated unsuccessfully with balloon compression. All patients had been refractory to antiepileptic drugs.

This procedure was well tolerated in all patients. Five patients experienced complete pain relief lasting more than 30 days, and 2 patients were completely unresponsive. No complications were observed.

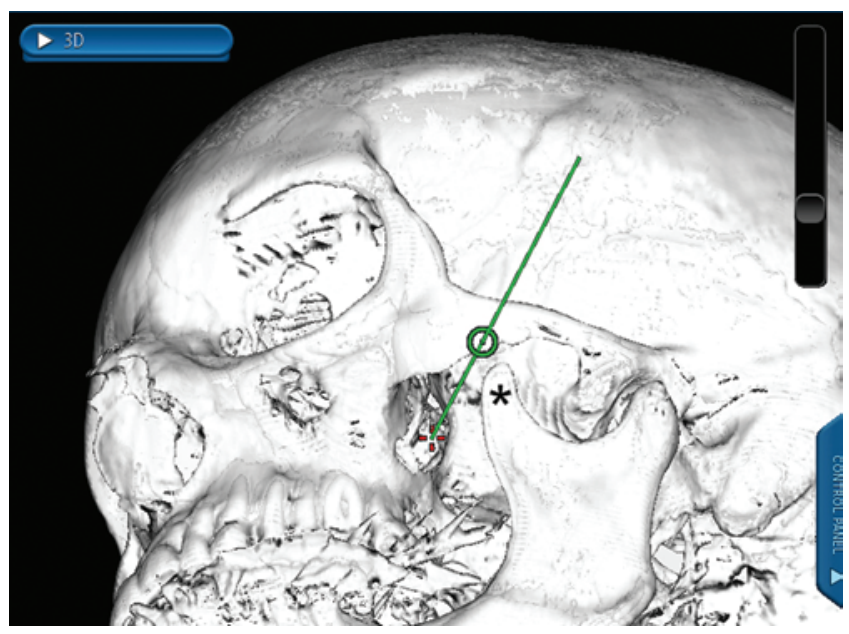

FIG. 2. Three-dimensional CT reconstruction showing the target in the PPF (red cross). The entry point (green circle) is chosen below the zygomatic arch and anterior to the coronoid process of the mandibular bone (asterisk). Figure is available in color online only.

\section{Discussion}

In 1908, Sluder ${ }^{7}$ was the first author to describe facial pain arising from the SPG and its treatment via phenol injection into the ganglion. Currently, the SPG is assumed to be involved in a great many types of facial pain, such as Sluder's neuralgia, ${ }^{4} \mathrm{TN},{ }^{4}$ persistent idiopathic facial pain, ${ }^{3}$ cluster headache, ${ }^{6}$ postherpetic TN, and other types of facial pain. ${ }^{4}$

Although GK treatment has been reported ${ }^{2}$ with promising results, this method is limited to those centers where a GK machine is available. Pain relief is also not immediate with $\mathrm{GK}$ treatment, often requiring up to several months to obtain some benefit. Today, the most common procedures are still the percutaneous ones: radiofrequency (RF) thermocoagulation ${ }^{8}$ or injections of glycerol/pure alcohol. ${ }^{4}$ Placing the tip of the needle as near to the SPG as possible is the key in all percutaneous procedures.

Although some authors have suggested the use of ultrasound echography to locate the PPF, ${ }^{5}$ followed by the injection of a large amount of local anesthetic that would reach the structures in PPF by diffusion, the best approach to avoid complications appears to be targeting the SPG precisely. The SPG lies deep in the PPF and is surrounded by the vidian nerve, the maxillary artery, and the maxillary nerve that gives 2 branches to the SPG to form the

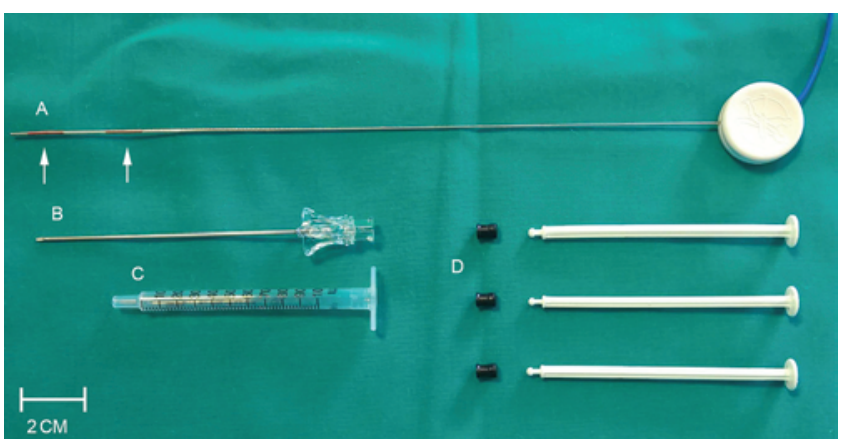

FIG. 3. Overview of the devices required to build a navigated needle. A: Magnetic stylet. The arrows indicate the 2 magnetic strips at the tip of the stylet. B: Spinal needle $(20 \mathrm{G})$. C: Insulin hypodermic syringe. D: Three rubber plunger tips from insulin hypodermic syringes. Figure is available in color online only. 


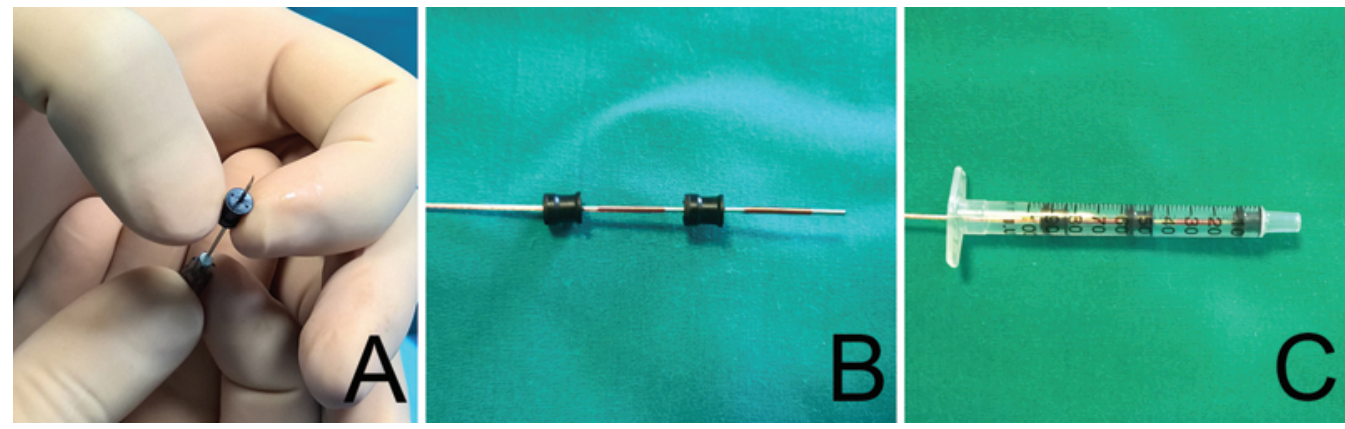

FIG. 4. Photographs showing the steps to firmly place the stylet inside the syringe. A: Two of the 3 plunger tips are pierced centrally. B: The magnetic stylet is inserted through the 2 pierced plunger tips. C: The third plunger tip is placed into the syringe to prevent the exit of the stylet from the syringe. Figure is available in color online only.

greater palatine nerve and the nasal palatine nerve (Fig. 7). A lesion of the last 2 can lead to permanent hypoesthesia of the hemipalate and/or the nasal mucosa.

Another factor to take into account is the SPG's small diameter $(4 \mathrm{~mm})$, considering that it is mandatory to place the needle tip as close as possible to the ganglion to avoid lesions to the surrounding structures. The infrazygomatic route is usually the approach chosen by most authors, and to achieve the most precise results the procedure is performed with the aid of fluoroscopy or CT.

Sanders et al. ${ }^{8}$ described an RF lesion of the SPG, using fluoroscopy to roughly reach its position, and then localizing the ganglion through the use of an electrical stimulus $(0.2-1 \mathrm{~V})$ and finally observing the patient's sensations: paresthesia of the nose is the desired outcome, while conversely paresthesia of the cheek/upper lip or in the palate indicates that the cannula is misplaced and an adjustment is required. However this procedure is time-consuming and quite uncomfortable for the patient.

The use of a CT-guided procedure has been reported by Kastler et al. ${ }^{4}$ They used a reference scan made at the level of the zygomatic arch and then inserted a needle below the arch to the front orifice of the pterygoid canal (also called the vidian canal), which they used as a reference point. However, this could lead to a misplacement of the needle.

Alvernia et al. ${ }^{2}$ showed very clearly that the SPG ganglion lies between the vidian canal and the foramen rotundum on cadaveric specimens. They easily identified these 2 reference points on coronal CT scan reconstructions, and with some difficulty on sagittal reconstructions. They were not recognizable, however, on axial images. To use a coronal reconstruction, the best way to plan a percuta-

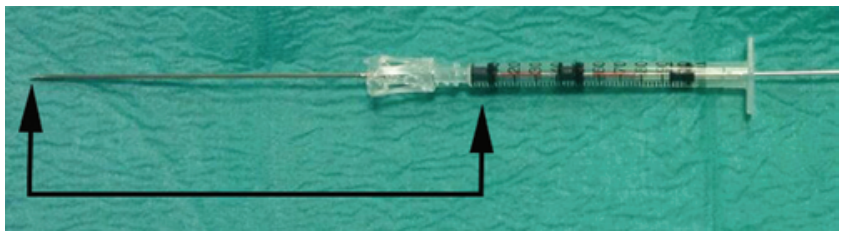

FIG. 5. Photograph of the navigated needle. The $20-\mathrm{G}$ spinal needle is connected to the syringe and is the direct continuation of the magnetic stylet. To navigate the needle's tip, the distance between the 2 arrows has to be inserted as the offset into the navigator. We usually do this by placing the needle on a bony surface such as the zygomatic arch, and then moving the offset upward until the tool's tip shown on the monitor reaches the skin. Figure is available in color online only. neous procedure to the SPG is certainly a navigated one. Navigation systems are currently available that allow the neuronavigation of a needle for percutaneous procedures of any caliber and length. However, these systems rely on optical references that require 3-point head fixation and general anesthesia.

Despite this being a neurosurgical era where neuronavigation is almost everywhere, there are to our knowledge no reports of percutaneous procedures conducted with the aid of neuronavigation, likely for the reason stated above. Furthermore, for this particular procedure there is no magnetic neuronavigated needle available that could render 3-point head fixation unnecessary. Medtronic makes a magnetic stylet that fits perfectly into a $14-\mathrm{G}$ needle, making it possible, for example, to perform a neuronavigated percutaneous balloon compression of the Gasser ganglion. This caliber, however, is too large for a procedure that does not require space for a Foley balloon to pass through. We used a $20-\mathrm{G}$ needle, and to navigate it we needed to build a device whereby the needle was the continuation of the line passing through the 2 magnetic strips placed at the tip of the stylet. That line is crucial because it allows the navigator to record the position of the stylet and its direction. The stylet itself can be bent at every point except between the 2 strips, due to the reason stated above.

Blocking the stylet inside a syringe with the plunger tips allows for the needle to be connected on the same plane as the stylet. The distance between the tip of the stylet and the tip of the needle is set as the offset, and from that point on the navigator will show the needle's tip as the new tool's tip on the screen, making it possible to navigate the needle. The assembly is sturdy and comfortable to handle for the duration of the procedure. The "target guidance" function makes it possible to reach the SPG safely, and in a matter of seconds. This method is probably not very elegant, but it demonstrates that a neuronavigated percutaneous approach to the SPG is feasible and reproducible.

Compared with other percutaneous procedures, the neuronavigated percutaneous approach allows the medical team to plan the needle's route on coronal and sagittal reconstructions. The planning is also performed preoperatively, which shortens the procedure time and the patient's discomfort. Using a magnetic reference system allows the operator to conduct the procedure under local anesthesia without using the 3-point head holder.

Another benefit to consider is that once the patient's CT 

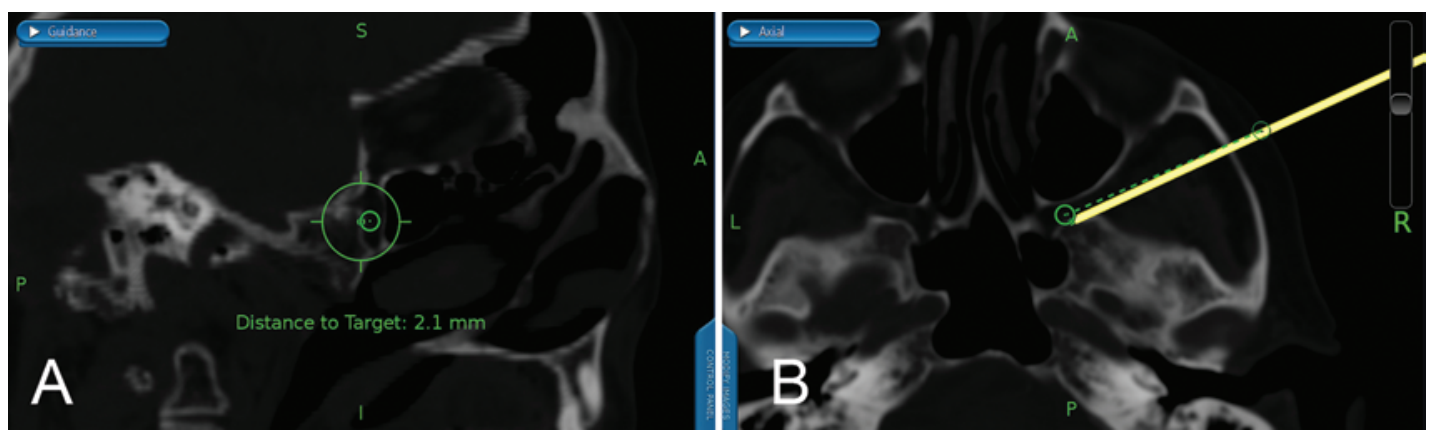

FIG. 6. Screenshots of the navigator's screen during the procedure. A: The guidance view on CT reconstructions allows one to correct the trajectory continuously. The tool (large green circle) can be easily aligned to the target (small green circle). The distance from the target is shown on screen. B: The same misalignment shown on the guidance view is represented on an axial plane (dashed line = planned trajectory, yellow line = actual needle position). Figure is available in color online only.

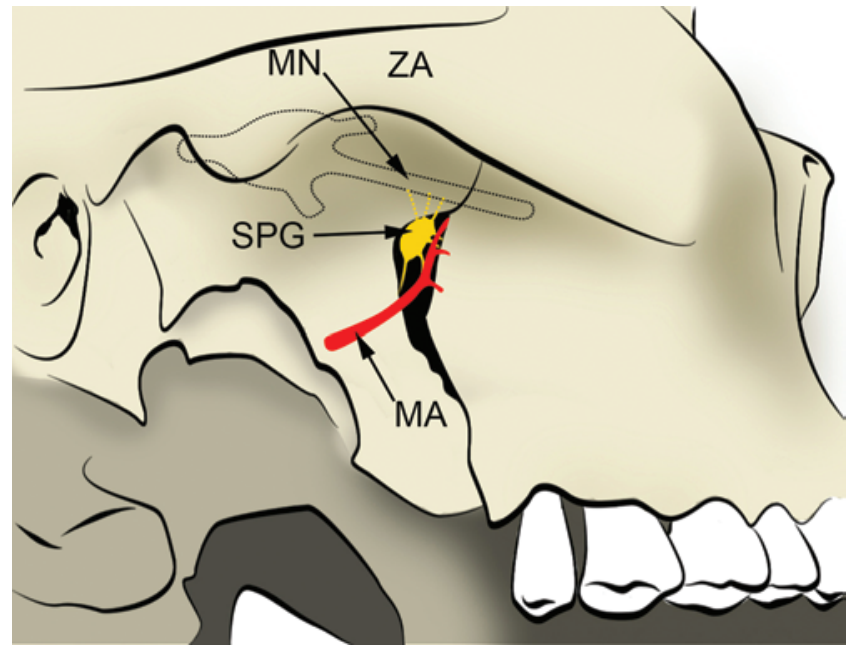

FIG. 7. Artist representation showing the position of the SPG within the PPF and its relationships with the surrounding structures. $M A=$ maxillary artery; $\mathrm{MN}=$ maxillary nerve; $\mathrm{ZA}=$ zygomatic arch. Copyright Nicola Benedetto. Published with permission. Figure is available in color online only.

scan is acquired, it will be available for future procedures. Most authors agree that these percutaneous procedures can be performed more than once, the clear advantage being that both doctors and patients are spared exposure to ionizing radiation.

\section{Conclusions}

This is the first report of a neuronavigated percutaneous approach to the SPG. It demonstrates that the procedure is feasible and reproducible. With the creation of a proper navigated needle, this could become a widespread procedure.

\section{Acknowledgments}

We thank Ms. Juliet Strachan for English revision and Mr. Michele Arrighi for technical advice.

\section{References}

1. Al-Qudah M: Endoscopic sphenopalatine ganglion blockade efficacy in pain control after endoscopic sinus surgery. Int Forum Allergy Rhinol [epub ahead of print], 2015

2. Alvernia JE, Spomar DG, Olivero WC: A computed tomography scan and anatomical cadaveric study of the pterygopalatine ganglion for use in Gamma Knife treatment of cluster headache. J Neurosurg 107:805-808, 2007

3. Cepero R, Miller RH, Bressler KL: Long-term results of sphenopalatine ganglioneurectomy for facial pain. Am J Otolaryngol 8:171-174, 1987

4. Kastler A, Cadel G, Comte A, Gory G, Piccand V, Tavernier L, et al: Alcohol percutaneous neurolysis of the sphenopalatine ganglion in the management of refractory cranio-facial pain. Neuroradiology 56:589-596, 2014

5. Nader A, Kendall MC, De Oliveria GS, Chen JQ, Vanderby $\mathrm{B}$, Rosenow JM, et al: Ultrasound-guided trigeminal nerve block via the pterygopalatine fossa: an effective treatment for trigeminal neuralgia and atypical facial pain. Pain Physician 16:E537-E545, 2013

6. Schytz HW, Barløse M, Guo S, Selb J, Caparso A, Jensen $\mathrm{R}$, et al: Experimental activation of the sphenopalatine ganglion provokes cluster-like attacks in humans. Cephalalgia 33:831-841, 2013

7. Sluder G: Role of the sphenopalatine (Meckel's) ganglion in nasal headaches. N Y State J Med 87:989-990, 1908

8. Sanders M, Zuurmond WWA: Efficacy of sphenopalatine ganglion blockade in 66 patients suffering from cluster headache: a 12- to 70-month follow-up evaluation. J Neurosurg 87:876-880, 1997

\section{Disclosures}

The authors report no conflict of interest concerning the materials or methods used in this study or the findings specified in this paper.

\section{Author Contributions}

Conception and design: both authors. Drafting the article: Benedetto. Critically revising the article: Perrini.

\section{Correspondence}

Nicola Benedetto, Neurosurgical Department, Azienda Ospedaliero Universitaria Pisana-AOUP, Via Paradisa 2, Pisa 56127, Italy. email: n.benedetto@ao-pisa.toscana.it. 